\title{
Practical Model Reference Robust Control for Pneumatic
}

\section{Servo System ${ }^{\dagger}$}

\author{
Junbo SoNG* , Kazunori KADOWAKI* and Yoshihisa ISHIDA*
}

\begin{abstract}
A practical control scheme of model reference robust control (MRRC) for pneumatic servo system is introduced in this paper. As an important driving element, the pneumatic cylinders are widely used in industrial applications. It is well known the control problem of pneumatic cylinder becomes complex in varying load, travel and environment temperature, because it contains unknown parameters, possible nonlinear uncertainties and additive bound disturbances. The proposed control scheme is essential to achieve the robust stability and good performance for time-varying and nonlinear systems. The controller requires only measured input and output signals of system, rather than the full state feedback. In order to suit for practical application the neural control parameters estimator is led into the controller. Compared with MRAC, it makes the controller become simple.
\end{abstract}

Key Words : model reference robust control, pneumatic cylinder, nonlinear plant, neural network, external disturbance

\section{Introduction}

Robust feedback controllers are the most common control schemes used for the control of nonlinear plant with uncertain dynamics. Their popularity is due to the simplicity of their structures as well as the familiarity of designers with feedback control theory.

In most robust feedback control schemes ${ }^{4), 5)}$, the authors either use state feedback to place the poles sufficiently far in the lefthalf-plane, or insert a feedback compensator in the closed loop feedback control system to cancel the effects of system uncertainties and guarantee the stability of the closed loop system. However, for the most of these schemes, the transient error response of the closed loop system can not be specified directly in the design. Therefore, the design of the high quality tracking controller which can guarantee good system performance is still a challenging topic in the servo control fields ${ }^{6), 77,8)}$.

It is worth mentioning that other nonlinear controllers such as high-gain control and sliding mode control are also based on Lyapunov designs and have similar robustness properties except that high-gain control may require excessively large control effect and sliding mode control is inherently discontinuous.

$\mathrm{Qu}$ has put forward the conception of MRRC ${ }^{1), 2), 3)}$, and given the controller design method from minmax Lyapunov design for continuous control system. Qu's results make us have the capability to handle with the control problem as MRAC using

\footnotetext{
$\dagger$ Presented in ICONIP'95(1995 $\cdot 10)$

* Department of Electronics and Communication, Meiji University, Tama-ku, Kawasaki

(Received September 6, 1995)

(Revised June 11, 1997)
}

robust control theory.

In this paper, to apply MRRC to practical control problems and improve the control property of pneumatic servo system, we lead a neural estimator into the controller which can estimate the control parameters of system and update the parameters error bounds used in robust controller on-line. It improves the output tracking accuracy and system robustness. Then we put forward the control model for plant with nonlinear uncertainties, which makes MRRC controller suit for control problem of pneumatic servo system. The experiments for practical model can prove the effectiveness of this control scheme.

This paper is organized as follows: Section 2 contains the configuration of pneumatic servo system. In Section 3 the MRRC is introduced simply. The system controller and control parameters estimator are given in Section 4. The results of simulation and experiment are shown in Section 5. In Section 6, we discuss the conclusions of this control scheme.

\section{Configuration of pneumatic servo system}

Fig. 1 shows the configuration of the pneumatic servo system. The system is primarily composed of the pneumatic cylinder, a 32 bit personal computer with an $80486 \mathrm{CPU}$ and an associated arithmetic coprocessor, two 12-bit D/A converters, two servo amplifiers of the voltage-current transformation type, and two electro-pneumatic proportional valves.

The pneumatic cylinder (CA1BQ63-300: SMC Corporation) is a low friction type. Its minimum pressure is $0.01 \mathrm{MPa}$, the inside diameter is $\phi 63$, and the stroke is $300 \mathrm{~mm}$. A load mass $10 \mathrm{~kg}$ is usually connected to the tip of a position rod. The electropneumatic proportional valve is a pressure proportional control 
valve(VEP3120-1 : SMC Corporation) of 3 port type. The

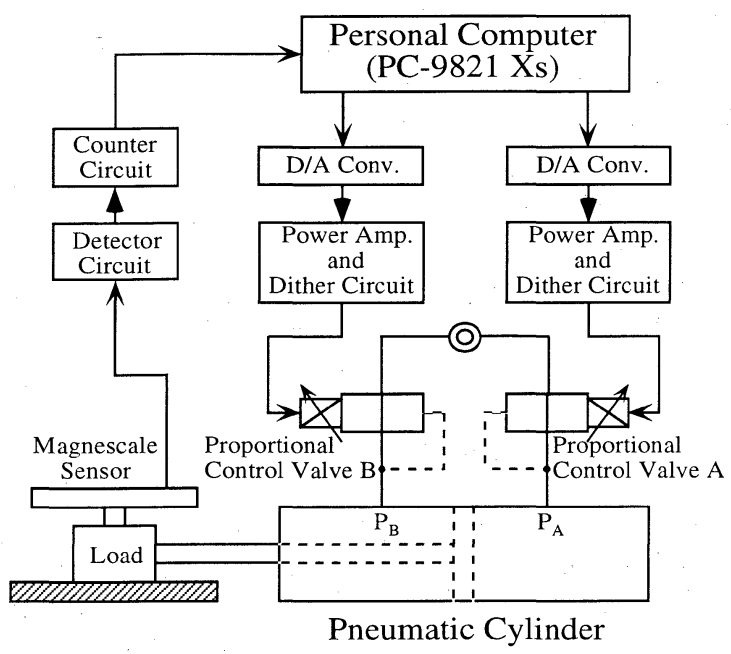

Fig. 1 Configuration of the pneumatic servo system

position measurement is carried out by a magnetic scale sensor whose resolving power is $10 \mu \mathrm{m}$ (SR10-100A : SONY Corporation).

If we define the system input as $p(t)=P_{A}-P_{B}$ and the position of piston as $y(t)$, usually, we can describe the dynamic equation of pneumatic cylinder as follows:

$$
\ddot{y}(t)+\frac{D}{M} \dot{y}(t)=\frac{A}{M}[u(t)+d(y, \dot{y}, t)]
$$

where

$$
\begin{aligned}
& A: \text { area of piston } \\
& D: \text { sticky friction parameter } \\
& M: \text { load mass }
\end{aligned}
$$

$d(y, \dot{y}, t):$ the lumped disturbance and uncertainty caused by the uncertain friction dynamics, air compression dynamics and unknown external disturbance.

As the uncertain friction dynamics is always bounded, for the further analysis, we assume the existence of a continuous, nonnegative, known function $\rho(y, t)$ such that

$$
|d(y, \dot{y}, t)|<\rho(y, t)
$$

\section{Model reference robust control}

According to Qu's model reference robust control theory, we can describe the plant as (3)

$$
\begin{aligned}
y^{(n)}(t)+ & a_{p 1} y^{(n-1)}(t)+\cdots+a_{p n} y(t)=b_{p 0}\left[u^{(m)}(t)\right. \\
& \left.+b_{p 1} u^{(m-1)}(t)+\cdots+b_{p m} u(t)+d_{1}(y, t)\right]
\end{aligned}
$$

where $m \leq n, d_{l}(y, t)$ is the external disturbance and can be bounded by a nonlinear function defined explicitly by the output $y$ and time $t$. When the disturbance is absent we have the transfer function relationship as

$$
\begin{aligned}
\left.\frac{Y(s)}{U(s)}\right|_{d_{1}=0} & =b_{p 0} \frac{s^{m}+b_{p 1} s^{m-1}+\cdots+b_{p m}}{s^{n}+a_{p 1} s^{n-1}+\cdots+a_{p n}} \\
& \triangleq k_{p} \frac{B_{p}(s)}{A_{p}(s)} \triangleq G_{p}(s)
\end{aligned}
$$

The control problem investigated in MRRC is the model following problem for which the output of the plant (3) tracks the output of a reference model for any given reference signal $r(t)$ in the presence of disturbance $d_{1}(y, t)$. The reference model is described by the following transfer function

$$
\begin{aligned}
\frac{Y_{m}(s)}{R(s)} & =b_{m 0} \frac{s^{m}+b_{m 1} s^{m-1}+\cdots+b_{m p m}}{s^{n}+a_{m 1} s^{n-1}+\cdots+a_{m n}} \\
& \triangleq k_{m} \frac{B_{m}(s)}{A_{m}(s)} \triangleq G_{m}(s)
\end{aligned}
$$

where $Y_{m}(s)$ and $R(s)$ are Laplace transforms of $y_{m}(t)$ and $r(t)$, respectively, $k_{m}>0$ and $r(t)$ is a bounded reference input. It is assumed that both $A_{m}(s)$ and $B_{m}(s)$ are coprime Hurwitz polynomials.

To achieve perfect tracking using only input and output data, a strictly positive real (SPR) transfer function is generated from the transfer function of the reference model. Define

$$
\bar{G}_{m}(s)=G_{m}(s) \alpha(s) \triangleq k_{m} \frac{\bar{B}_{m}(s)}{A_{m}(s)}
$$

where $\alpha(s)$ is a monic, Hurwitz polynomial in $s$ of degree $m-n$ 1 , and $G_{m}(s)$ is the reference model. If $G_{m}(s)$ has relative degree larger than the relative degree of $G_{p}(s), \alpha(s)$ is chosen such that $\bar{G}_{m}(s)$ has relative degree one. It is assumed that $\bar{G}_{m}(s)$ is SPR

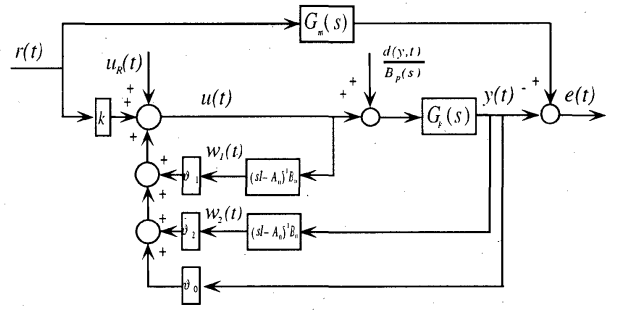

Fig. 2 Block diagram of model reference robust control

The block diagram of MRRC is shown in Fig. 2. According to it, the following system variable can be defined.

I: Auxiliary signal $w(t)$

$$
w(t)=\left[r(t), y(t), w_{1}^{T}(t) w_{2}^{T}(t)\right]^{T}
$$

where

$$
\begin{aligned}
& \dot{w}_{1}(t)=A_{0} w_{1}(t)+B_{0} u(t) \\
& \dot{w}_{2}(t)=A_{0} w_{2}(t)+B_{0} y(t)
\end{aligned}
$$

and $w_{1}(t) \in R^{(n-1)}, w_{2}(t) \in R^{(n-1)}$ are state vectors, $A_{0} \in R^{(n-1) \times(n-1)}$ is a matrix such that $\operatorname{det}\left[s I-A_{0}\right]=\overline{B_{m}}(s)$ and $B_{0} \in R^{(n-1)}$ is a constant vector such that the pair $\left(A_{0}, B_{0}\right)$ is controllable.

II: The output tracking error $e(t)$

$$
e(t)=y_{m}(t)-y(t)
$$

III: The states of the controllable canonical realization of the transfer function $1 / \alpha(s)$ can be defined. That is, if

$$
\alpha(s)=s^{l}+\alpha_{1} s^{l-1}+\cdots+\alpha_{i} s^{l-i}+\cdots+\alpha_{l}
$$

we define 


$$
\begin{aligned}
z_{1} & =u_{R} / \alpha_{1} \\
\dot{z}_{i} & =z_{i+1} \quad i=1,2, \cdots, l-1 \\
\dot{z}_{l} & =-\alpha_{1} z_{1}-\cdots-\alpha_{r} s^{l-i}+u_{R} \\
& =-\alpha_{1} z_{1}-\cdots-\alpha_{s} s^{l-i}+u-\vartheta^{T} w(t)
\end{aligned}
$$

where $l=n-m-1$.

IV: The auxiliary filter vector $\bar{w}(t)$

$$
\bar{w}(t)=\left[\dot{r}(t), \bar{y}(t), \bar{w}_{1}^{T}(t) \bar{w}_{2}^{T}(t)\right]^{T}
$$

where

$$
\begin{array}{ll}
\dot{r}(t)=\frac{1}{\alpha(s)} r(t), & \bar{y}(t)=\frac{1}{\alpha(s)} y(t) \\
\bar{w}_{1}(t)=\frac{1}{\alpha(s)} w_{1}(t), & \bar{w}_{2}(t)=\frac{1}{\alpha(s)} w_{2}(t)
\end{array}
$$

$\mathrm{V}$ : Control parameter error vector $\bar{\vartheta}$

$$
\bar{\vartheta}=\vartheta^{*}-\vartheta
$$

where $\vartheta^{*}$ is the desired controller parameter, and $\vartheta$ is an arbitrary estimate of $\vartheta^{*}$.

VI: Let $\psi(y, t)$ be a continuous function. Then $B N D(\|\psi(y, t)\|)$ is a known, continuous and nonnegative function that bounds the magnitude ( or Euclidean norm ) of $\psi(y, t)$. That is, for all $(y, t) \in R \times R^{+}$

$$
\|\psi(y, t)\| \leq B N D(\|\psi(y, t)\|)
$$

Thus the auxiliary control variables can be defined as follows:

$$
v_{1}=\frac{\mu_{1}(e, y, u, t)\left|\mu_{1}(e, y, u, t)\right|}{2\left(\left|\mu_{1}(e, y, u, t)\right|^{2}+\delta_{1}\right)} g_{1}(y, u, t)
$$

where $\mu_{1}(e, y, u, t)=e(t) g_{1}(y, u, t)$ and

$$
\begin{aligned}
g_{1}(y, u, t)= & 2 B N D(\|\bar{\vartheta}\|) \times\|\bar{w}(t)\| \\
& +2 B N D\left(\left|\frac{1}{\alpha(s)} \times\left(1-\frac{D_{1}(s)}{\bar{B}_{m}(s)}\right) \times d_{1}(y, t)\right|\right)
\end{aligned}
$$

The following $l$ mappings are then defined by

$$
\begin{aligned}
v_{i} & =e+v_{i-2}-z_{i-2}+v_{i-1}-z_{i-1} \\
& +\frac{\mu_{i}(e, y, u, t)\left|\mu_{i}(e, y, u, t)\right|}{2\left(\left|\mu_{i}(e, y, u, t)\right|^{2}+\delta_{i}\right)} g_{i}(y, u, t)
\end{aligned}
$$

and

$$
\begin{aligned}
v_{l+1}= & \alpha_{1} z_{l}+\alpha_{2} z_{l-2}+\cdots+\alpha_{l} z_{1}+v_{l-1}-z_{l-1}+v_{l}-z_{l} \\
& +\frac{\mu_{l+1}(e, y, u, t)\left|\mu_{l+1}(e, y, u, t)\right|}{2\left(\left|\mu_{l+1}(e, y, u, t)\right|^{2}+\delta_{l+1}\right)} g_{l+1}(y, u, t)
\end{aligned}
$$

For $i=2,3, \ldots, l$ with

$$
\begin{aligned}
& g_{i}(y, u, t)=2 B N D\left(\left|\dot{v}_{i-1}\right|\right) \\
& \mu_{i}(e, y, u, t)=\left(v_{i-1}-z_{i-1}\right) g_{i}(y, u, t) \\
& g_{l+1}(y, u, t)=2 B N D\left(\left|\dot{v}_{l}\right|\right) \\
& \mu_{l+1}(e, y, u, t)=\left(v_{l}-z_{l}\right) g_{l+1}(y, u, t)
\end{aligned}
$$

Quotation: If we choose the robust control $u(t)$ as

$$
u(t)=\vartheta T_{W}(t)+u_{R}(t) ; u_{R}(t)=v_{l+1}(t)
$$

the plant output tracking error $e(t)$ is globally uniformly ultimately bounded. That is, as time approaches infinity, the magnitude of the output tracking error becomes no larger than a constant $\delta$ which can be made arbitrarily small by choosing the design parameters $\delta_{i}$ small enough. Furthermore, the robust control $u(t)$ is continuous and globally, uniformly bounded ${ }^{2)}$.

\section{Practical MRRC for pneumatic servo system}

On the basis of the dynamical model of pneumatic cylinder described in Eq. (1), it can be thought as a classical second order control element, take the lumped disturbance, nonlinear factor and uncertainty described by a nonlinear unknown function $d(y, \dot{y}, t)$ into account, we can design the practical MRRC controller for pneumatic servo system.

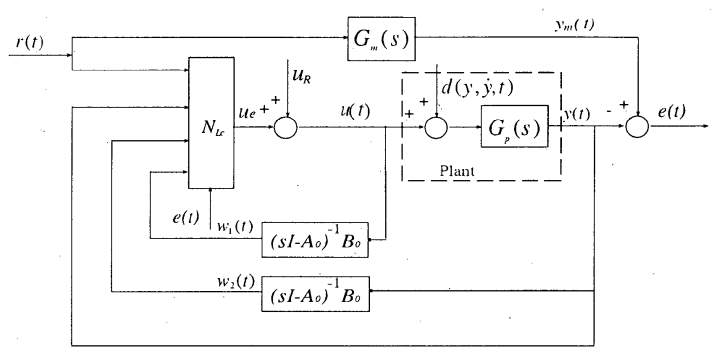

Fig. 3 Block diagram of the practical MRRC

\subsection{Definitions of plant and reference model}

According to the dynamical model of pneumatic cylinder described in Eq. (1), we can rewrite the linear part of Eq. (1) as follows:

$$
G_{p}(s)=k_{p} B_{p}(s) / A_{p}(s)=k_{p} /\left(s^{2}+a_{p 1} s+a_{p 2}\right)
$$

where $k_{p}=A / M, k_{p}>0, a_{p 1}=D / M$ and $a_{p 2}=0$. On the other hand, the nonliner part can be described by the lumped disturbance and uncertainty $d(y, \dot{y}, t)$.

The reference model can be defined as

$$
G_{m}(s)=k_{m} B_{m}(s) / A_{m}(s)=k_{m} /\left(s^{2}+a_{m l} s+a_{m 2}\right)
$$

In the case that $G_{m}(s)$ is not SPR, let $\alpha(s)=\mathrm{s}+\alpha_{0}$ and assume that $\alpha(s) G_{m}(s)$ is SPR.

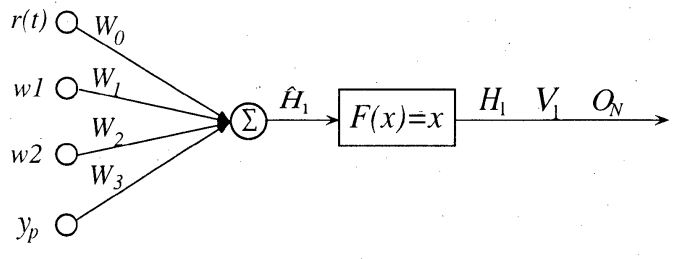

Fig. 4 Block diagram of control parameters estimator

\subsection{On-line control parameters estimator}

It is noted in 2) that the tracking error can be made smaller by choosing a small value of the design parameters $\delta_{i}$. If $\delta_{i}$ is too small, however, the output of the robust controller may change rapidly and has a large magnitude. Since the parameters error bounds and the lumped disturbance and uncertainty bound function are usually conservative, the design parameter $\delta_{i}$ can not be set too small. In this paper, in order to overcome this 
shortage and improve the control performance, we lead the controller estimator to calculate the parameter bounds into the practical controller.

Fig. 4 shows the block diagram of control parameters estimator formed by a linear neural network, where $W_{i}$ is the coupling coefficient between an input layer node and a hidden layer node $V_{1}$ is the coupling coefficient between a hidden layer node and an output layer node. $O_{N}$ is the output of neural network. If we define

$$
\vartheta(t)=V_{l}(t)\left[W_{0}(t), W_{1}(t), W_{2}(t), W_{3}(t)\right]^{T}
$$

we have

$$
u_{e}(t)=O_{N}(t)=\vartheta^{T}(t) w(t)
$$

where $\vartheta(t)$ is the estimate of $\vartheta^{*}$ in instant $t$. Network can be trained by $\mathrm{BP}$ algorithm 8 ) as follows:

$$
\begin{aligned}
& V_{1}(\mathrm{t})=V_{1}(t-1)+\Delta V_{1}(t) \\
& W_{\mathrm{i}}(\mathrm{t})=W_{\mathrm{i}}(t-1)+\Delta W_{\mathrm{i}}(t)
\end{aligned}
$$

where

$$
\begin{aligned}
& \Delta V_{1}=-\eta\left(\partial E / \partial V_{1}\right) \\
& \Delta W_{i}=-\eta\left(\partial E / \partial V_{i}\right.
\end{aligned}
$$

and $E$ is the auxiliary error, it can be defined as

$$
E(t)=\frac{1}{2}\left[y(t)-y_{m}(t)\right]^{2}
$$

From Fig. 4, taking control parameters estimator into account, we can rewrite the control of closed loop system as

$$
u(t)=u_{\mathrm{R}}(t)+u_{\mathrm{e}}(t)
$$

\subsection{Design of practical MRRC controller}

On the basis of definitions of pneumatic cylinder and reference model in Eqs.(21) and (22) we can design the MRRC controller as follows:

Step 1:

Define system variables as

$$
\begin{aligned}
& A_{0}=-\alpha_{0}, B_{0}=1, D_{1}(s)=\vartheta_{1}, \\
& D_{2}(s)=\vartheta_{0}\left(s+\alpha_{0}\right)+\vartheta_{2} \\
& \vartheta=\left[k, \vartheta_{0}, \vartheta_{1}, \vartheta 2\right]^{T}
\end{aligned}
$$

where $\vartheta$ is the arbitrary estimate of $\vartheta^{*}$. It is estimated by control parameters estimator described in 4.2 and used to bound $\bar{\vartheta}=\vartheta^{*}-\vartheta$ in each instant, where $\vartheta^{*}=\left[k^{*}, \vartheta_{0}^{*}, \vartheta^{*}, \vartheta^{*}\right]^{T}$. For the estimate of the parameter bounds, we assume that $\operatorname{sign}(k *)$ and $\operatorname{sign}\left[\vartheta_{i}^{*}\right]$ are known in this paper.

Step 2:

Generate, if desired, the auxiliary signals

$$
\begin{array}{ll}
\dot{w}_{1}=-\alpha_{0} w_{1}+u & \dot{w}_{2}=-\alpha_{0} w_{2}+y \\
\dot{\bar{r}}=-\alpha_{0} \dot{r}+r & \dot{\bar{y}}=-\alpha_{0} \bar{y}+y \\
\dot{\bar{w}}_{1}=-\alpha_{0} \bar{w}_{1}+w_{1} & \dot{\bar{w}}_{2}=-\alpha_{0} \bar{w}_{2}+w_{2} \\
\dot{\bar{z}}=-\alpha_{0} \bar{z}+z &
\end{array}
$$

Step 3:

Determine the control from the recursive mapping

$$
\begin{aligned}
v_{1} & =\frac{\mu_{1}(e, y, u, t)\left|\mu_{1}(e, y, u, t)\right|}{2\left(\left|\mu_{1}(e, y, u, t)\right|^{2}+\delta_{1}\right)} g_{1}(y, u, t) \\
& =\frac{e|e| g_{1}^{3}}{2\left(e^{2} g_{1}^{2}+\delta_{1}\right)}
\end{aligned}
$$

where $\delta_{1}>0$ is a constant, and $\mu_{1}(e, y, u, t)=e g_{1}(y, u, t)$ and

$$
\begin{aligned}
g_{1}(y, u, t) \triangleq & B N D\left(\tilde{k}^{2}\right)+B N D\left(\tilde{r}^{2}\right)+B N D\left(\tilde{\vartheta}_{0}^{2}\right)+\bar{y}^{2}(t) \\
& +B N D\left(\widetilde{\vartheta}_{1}^{2}\right)+B N D\left(\widetilde{\vartheta}_{2}^{2}\right)+\bar{w}_{1}^{2}(t)+\bar{w}_{2}^{2}(t) \\
& +2 \int_{t_{0}}^{t} \exp \left[-\alpha_{0}(t-\tau)\right] \rho(y, t) d \tau+B N D\left(\vartheta_{1}\right) \\
& \times 2 \int_{t_{0}}^{t}(t-\tau) \exp \left(-\alpha_{0} t\right) \rho(y, t) d \tau
\end{aligned}
$$

Thus, we can rewrite the control of overall system as:

$$
\begin{aligned}
u(t) & =\vartheta^{T} w(t)+u_{R} \\
& =\vartheta^{T} w(t)+\alpha_{0} z(t)+e(t)+v_{1}(t)-z(t) \\
& +\frac{\mu_{2}(e, y, u, t)\left|\mu_{2}(e, y, u, t)\right|}{2\left(\left|\mu_{2}(e, y, u, t)\right|^{2}+\delta_{2}\right)} g_{2}(y, u, t)
\end{aligned}
$$

where $\delta_{2}>0$ is a constant,

$$
\begin{aligned}
& \mu_{2}(e, y, u, t)=\left(v_{1}-z\right) g_{2}(y, u, t) \\
& B N D[\tilde{k}(t)]=\left|k^{*}(0)-k(t)\right| \\
& B N D[\tilde{\vartheta}(t)]=\left|\vartheta_{i}^{*}(0)-\vartheta_{i}(t)\right| \text { for } i=0,1,2 \\
& g_{2}\left((y, u, t)=2 B N D\left(\dot{v}_{1}\right)\right.
\end{aligned}
$$

and then the parameters $k^{*}(0)$ and $\vartheta_{i}^{*}(0)$ can be selected such that

$$
\begin{aligned}
& \left.\operatorname{sign}\left[k^{*}(0)\right]=\operatorname{sign}\left[k^{*}\right], \mid k^{*}(0)\right]|\geq| k^{*} \mid \\
& \left.\operatorname{sign}\left[\vartheta_{i}^{*}(0)\right]=\operatorname{sign}\left[\vartheta_{i}^{*}\right], \mid \vartheta_{i}^{*}(0)\right]|\geq| \vartheta_{i}^{*} \mid \quad i=0,1,2
\end{aligned}
$$

To implement the MRRC, we have to find a bounding function for $\left|\dot{v}_{1}\right|$. It follows that

$$
\frac{d v_{1}}{d t}=\frac{\partial v_{1}}{\partial e} \frac{d e}{d t}+\frac{\partial v_{1}}{\partial g_{1}} \frac{d g_{1}}{d t}
$$

in which every term is well defined and can be easily bounded. It follows that

$$
\begin{aligned}
B N D(\dot{e}) \triangleq & B N D\left(b_{p 0}\right)\left(|z|+0.5 g_{1}(y, u, t)\right. \\
& \left.+\int_{t_{0}}^{t} h(t-\tau)\left[|z|+0.5 g_{1}(y, u, t)\right] d \tau\right)
\end{aligned}
$$

where $h(t)$ is the inverse Laplace transform of $1-s \bar{G}_{m}(s)$ and $\bar{G}_{m}(s)=\left(s+\alpha_{0}\right) G_{m}$. Thus $g_{2}(y, u, t)$ can be chosen as

$$
g_{2}(y, u, t)=\frac{1.5|\dot{e}| g_{1}^{3}}{e^{2} g_{1}^{2}+\delta_{1}} B N D(|\dot{e}|)+5\left|\frac{d g_{1}}{d t}\right|
$$

or, if the simpler expression is desirable

$$
g_{2}(y, u, t)=\frac{1.5}{\delta_{1}} g_{1}^{2} B N D(|\dot{e}|)+5\left|\frac{d g_{1}}{d t}\right|
$$

Note that the choice of bounding function is not unique. The triangle inequality can be used to determine different choices. Usually, the tighter the bounding functions, the better the performance of the robust controller. The parameters chosen in (40) and (42) are experience values. On the basis of simulation results, they can be adopted by the overwhelming majority second 
order plants.

Step 4:

If there is enough prior knowledge for plant, we can calculate the desired parameter vector $\vartheta^{*}$ as follows:

$$
\vartheta^{*}=\left[\begin{array}{c}
\vartheta_{0}^{*} \\
\vartheta_{1}^{*} \\
\vartheta_{2}^{*}
\end{array}\right]=\left[\begin{array}{c}
\left(a_{p 1} \alpha_{0}+a_{p 1}^{2}-a_{p 1} a_{m 1}+a_{p 2}-a_{m 2}-\alpha_{0} a_{m 1}\right) / k_{p} \\
a_{m 1}-a_{p 1} \\
\left(a_{p 1} a_{p 2}-a_{p 2} a_{m 2}-a_{p 1} \alpha_{0}^{2}-a_{p 1}^{2} \alpha_{0}+a_{p 1} a_{m 1} \alpha_{0}\right. \\
\left.+\alpha_{0}^{2} a_{m 1}\right) / k_{p p}
\end{array}\right]
$$

It can be used to initiate $\vartheta_{i}^{*}(0)$. It is necessary to note that the step 4 is not indispensable for the design of controller. $\vartheta_{i}^{*}(0)$ can also be set by the experiment results step by step.

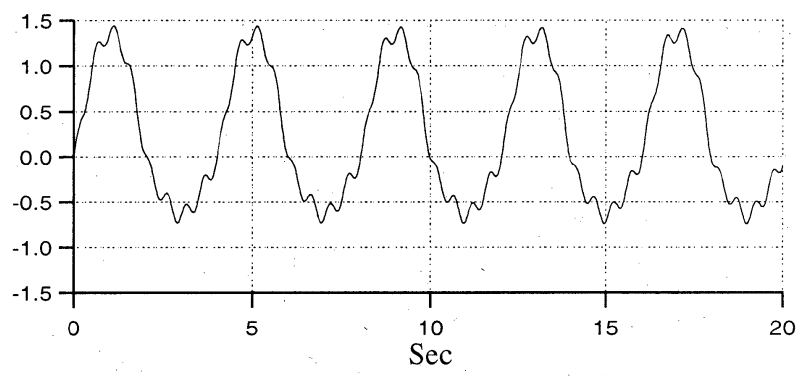

(a) Output response of plant with the P-controller

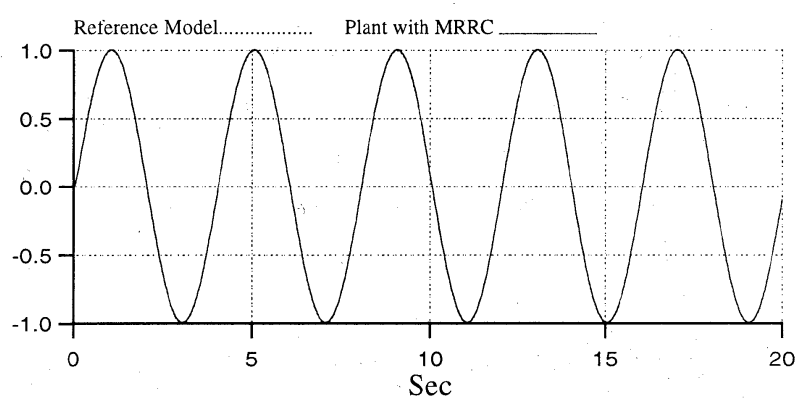

(b) Outputs of reference model and plant with the MRRC controller

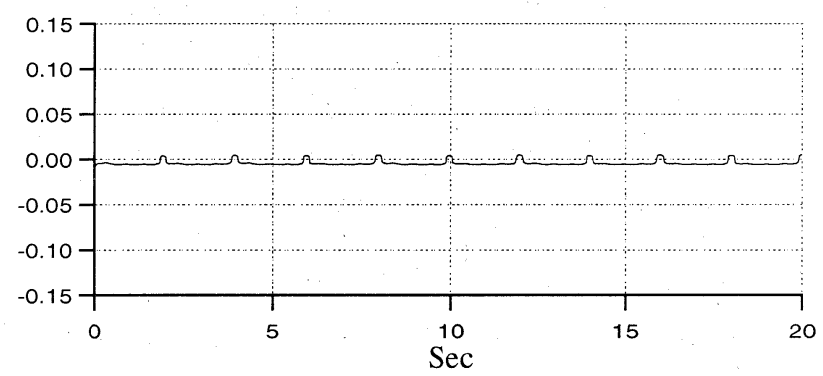

(c) Output track error $e(t)$

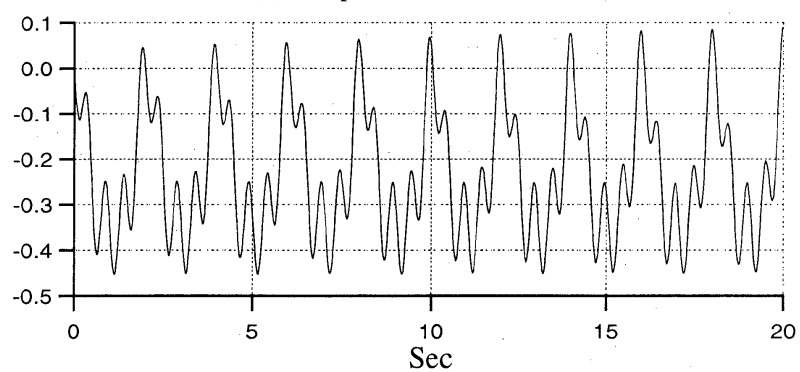

(d) Control signal $u(t)$ vs. time $t$

Fig. 5 The simulation results of the nonlinear plant with the MRRC controller

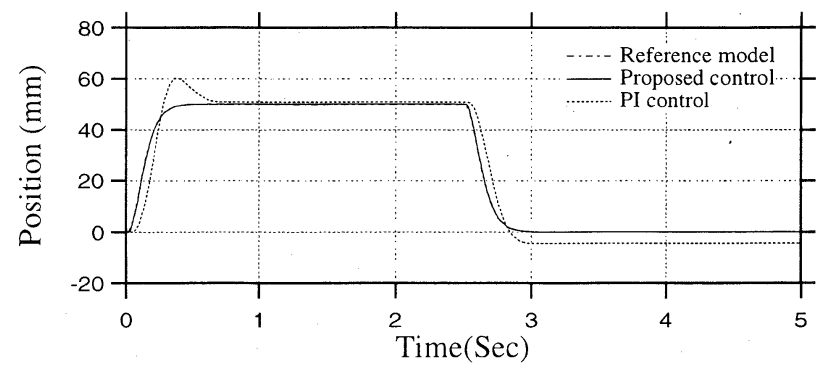

(a) Comparison between reference model and plant with the proposed controller and the PI-controller

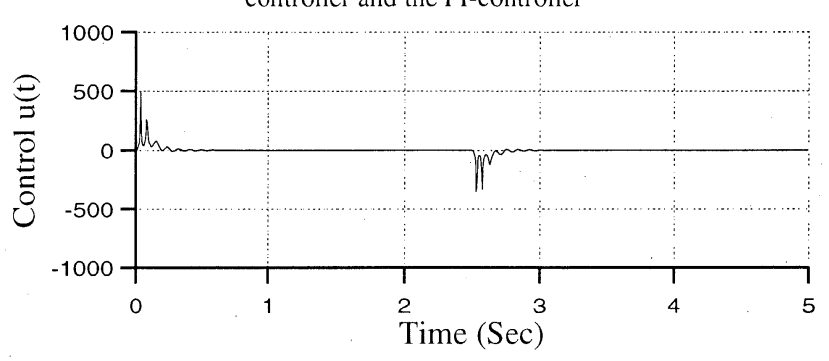

(b) Control signal $u(t)$ vs. time $t$

Fig. 6 The experiment results of pneumatic servo system with the MRRC controller $(\mathrm{M}=30 \mathrm{~kg})$

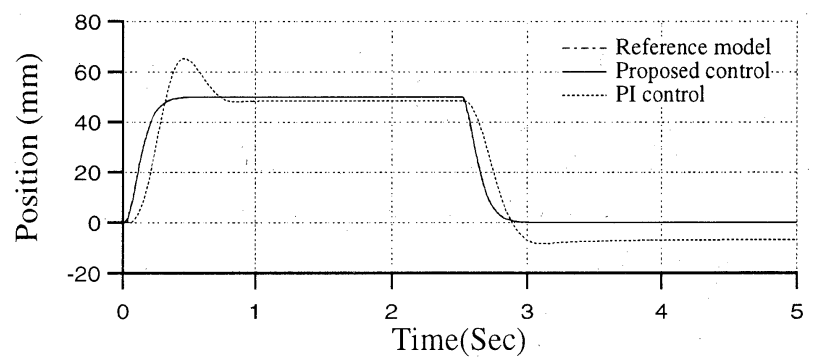

(a) Comparison between reference model and plant with the proposed controller and the PI-controller

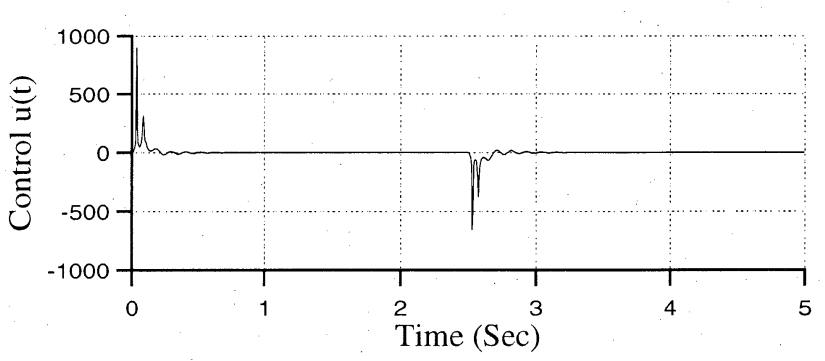

(b) Control signal $u(t)$ vs. time $t$

Fig. 7 The experiment results of pneumatic servo system with the MRRC controller $(M=60 \mathrm{~kg})$

\section{Results of Experiment}

\subsection{Results of simulation}

In order to check the effectiveness of the control scheme presented in Section 4, the simulation was carried out for a second order control plant as follows:

In the experiment, the reference model is defined as following transfer function 


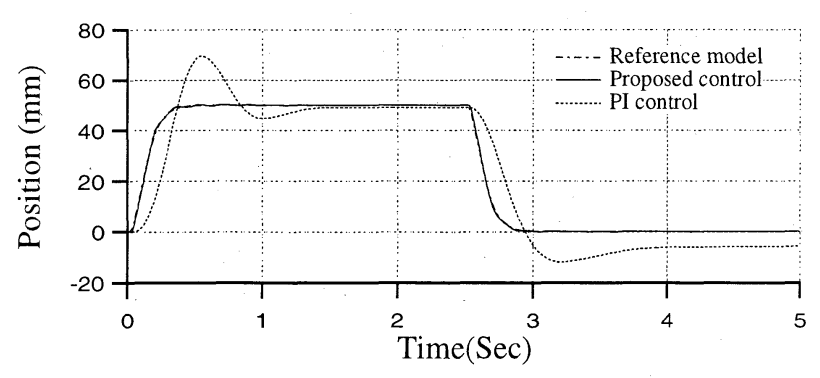

(a) Comparison between reference model and plant with the proposed controller and the PI-controller

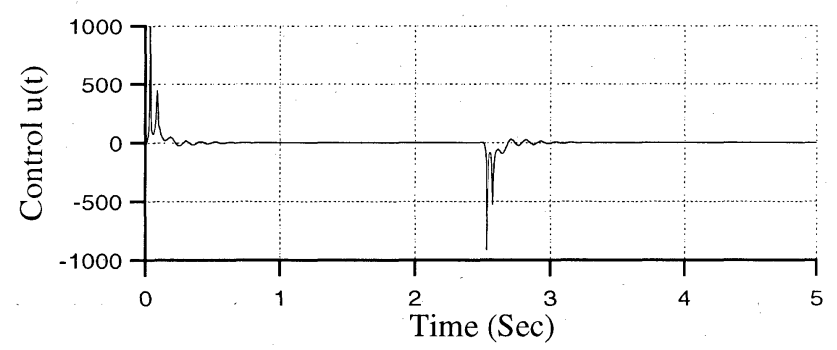

(b) Control signal $u(t)$ vs. time $t$

Fig. 8 The experiment results of pneumatic servo system with the MRRC controller $(\mathrm{M}=120 \mathrm{~kg})$

$$
G_{m}(\mathrm{~s})=100 /(s+10)^{2}
$$

and the reference input is $r(t)=\sin (0.5 \pi t)$.

The linear part of plant model is selected as the model of pneumatic cylinder with $30 \mathrm{~kg}$ load mass. It can be modeled by the least square algorithm as

$$
G_{p}(s)=\frac{1359}{s(s+70)}
$$

The lumped disturbance and uncertainty is set as

$$
d(y, \dot{y}, t)=\sin (3 t)+\frac{\sin (y)}{\left(1+y^{2}\right)}
$$

The $\eta$ of neural network $N_{L c}$ is set as 0.01 and the control parameters are set as follows:

$$
\begin{aligned}
& \alpha_{0}=1.0, \vartheta(0)=0.0, k^{*}(0)=15.0, \vartheta_{0}^{*}(0)=12.0, \vartheta_{1}^{*}(0)=5.0, \\
& \vartheta_{2}^{*}(0)=4.0, \rho(y, t)=1.0, B N D\left[k_{\mathrm{p}}(0)\right]=1500, \delta_{1}=\delta_{2}=0.2
\end{aligned}
$$

Simulation results are shown in Fig. 5.

Judged by the results of simulation for a nonlinear plant, we get the satisfied results using the control scheme described in Section 4 . It can not only assure the control precise, but also makes the system control continuous uniformly.

\subsection{Applying to pneumatic servo system}

The simulation results in 5.1 are excellent. In this section, we apply the proposed control scheme to pneumatic servo system described in Section 2. The parameters of controller are set as

$$
\begin{aligned}
& \alpha_{0}=1.0, \vartheta(0)=0.0, k^{*}(0)=15.0, \vartheta_{0}^{*}(0)=12.0, \vartheta_{1}^{*}(0)=5.0 \\
& \vartheta_{2}^{*}(0)=4.0, \rho(y, t)=1.0, B N D\left[k_{\mathrm{p}}(0)\right]=1500, \delta_{1}=\delta_{2}=0.2
\end{aligned}
$$

where the sampling time is set as $T_{s}=2 \mathrm{~ms}$, the reference input $r(t)=50 \mathrm{~mm}$. Learning rate $\eta$ of neural network $N_{L \mathrm{c}}$ is set by
0.01 . The same reference model is adopted as simulation. The experiment results in varying load mass are shown in Fig. 6, 7 and Fig. 8.

The experiment results in the case of $\mathrm{M}=30 \mathrm{~kg}$ illustrates that output response of system is excellent. The controller comes over the affection of stick friction and possible nonlinear uncertainties. It must be pointed that the dynamical error always exists because of stick friction. However, in practical application, perhaps, it is more important to ensure the good steady-state tracking precision. In our experiment the steady-state tracking error is always less than $10 \mu \mathrm{m}$.

It is well known that the model of plant will be changed along with varying of load mass. In order to get high control precision, generally, we have to tune the control parameters of controller repeatedly. However, we can still obtain satisfied control effects in difference load mass even though using the same control parameters with proposed control scheme.

The experimental results show that the dynamical tracking error becomes great along with increasing of load mass (peak error is less than $2 \mathrm{~mm}$ in the case of $M=120 \mathrm{~kg}$ and travel is $50 \mathrm{~mm}$ ), but the steady-state precision is still satisfied (the steadystate tracking error is not greater than $10 \mu \mathrm{m})$.

Compared with the results of plant with the PI-controller, we can think that the proposed control scheme is effective for the high precision pneumatic servo system.

\section{Conclusions}

We have proposed a control scheme for the pneumatic servo system. It can not only guarantee a good control precision but also come over nonlinear uncertainties contained in plant. It can make the pneumatic servo system with satisfied dynamical and steady responses in the case of the load mass varying, even if using the same control parameters. It shows excellent system performance, robustness and good applicability.

\section{Acknowledgment}

The authors would like to thank SMC Co., Ltd. for supporting this work.

\section{References}

1) Z. Qu: Model reference robust control of SISO systems with significant unmodeled dynamics, in Proc. 1993 Amer. Contr. Conf., San Francisco, CA. $255 / 259$ (1993)

2) Z. Qu: Model reference robust control of weakly nonminimum phase systems, in Proc. 32nd IEEE Conf. Decis. Contr., San Antonio, TX, WP-2 (1993)

3) Z. Qu: Global stabilization of nonlinear systems with a class of unmatched uncertainties, Syst. Contr. lett. vol.18, no. 3, 301/307 (1992)

4) Jabbari, F. : A noninterative method for the design of linear 
robust controllers, IEEE Trans, Automat. Contr., vol.35, 954/975 (1990)

5) Schmite Dorf, W. E., and Barmach, B. R. : Robust asymptotic tracking for linear system with unknown parameters, Automatics, vol. 22, 353/360 (1986)

6) Yeung, K. S., and Chen, Y. P. : A new controller design for manipulators using the theory of variable structure systems,

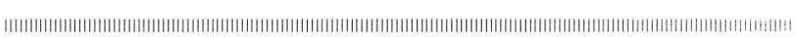

\section{[Authors' Profiles]}

\section{Junbo SoNG (Student Member)}

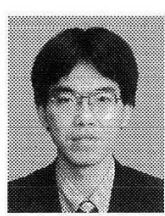

Junbo Song was born in Sandong, China in Feb. 15, 1966. He received the B.S. degree and MS. degree in Northwestern Polytechnic University and Beijing university of Aeronautics and Astronautics in 1987 and 1991 respectively.

Currently, Mr. Song is working toward the Ph.D. in Electronic engineering at Meiji University. His research interests include robust control, linear control theory, adaptive and learning systems, neural networks and their application to adaptive control problems.

Mr. Song is a student member of Society of Instrument and Control Engineers of Japan.

\section{Kazunori KADOWAKI}

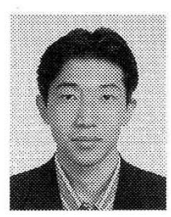

Kazunori Kadowaki received the B.S. degree and MS. degree in in Electronic engineering at Meiji University in 1995 and 1997 respectively. From April 1997, he has joined the Denso Corporation. His research interests include robust control, adaptive and learning systems, neural networks and their application to adaptive control problems.
IEEE Trans. Automat. Contr., vol. 33, 200/226 (1988)

7) M. W. Spong and M. Vidyasagar : Robust linear compensator design for nonlinear robotic control, IEEE J. Rob. Automat., vol. RA-3, 345/351 (1987)

8) K. Endo,Y. Ishida and T. Honda : Gain adjustment of I-PD control system using neural network, Trans. of IEE of Japan, vol.113-c, no.6, 408/416 (1993)

\section{Yoshihisa IsHIDA (Member)}

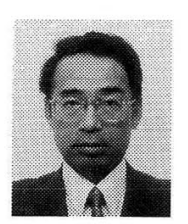

Prof. Yoshihisa Ishida received the B.S. degree and MS. and Ph. D. degree in Electronic engineering at Meiji University in 1970, 1972 and 1978 respectively. Currently, he is a professor in Department of Electronics and Communication, Faculty of Science \& Technology, Meiji University. His research interests include control theory, speech processing, neural network and their application to engineering problems.

Prof. Ishida is a member of IEEE, Society of Instrument and Control Engineers of Japan and the Institute of Electronics, information and Communication Engineers of Japan.

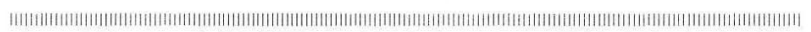

